\title{
Modeling Human Methamphetamine Exposure in Nonhuman Primates: Chronic Dosing in the Rhesus Macaque Leads to Behavioral and Physiological Abnormalities
}

\author{
Lisa J Madden', Claudia T Flynn', Michelle A Zandonatti', Meredith May', Loren H Parsons', \\ Simon N Katner', Steven J Henriksen' and Howard S Fox*, I \\ 'Department of Neuropharmacology, The Scripps Research Institute, La Jolla, CA, USA
}

\begin{abstract}
Acute high dose methamphetamine (METH) dosing regimens are frequently used in animal studies, however, these regimens can lead to considerable toxicity and even death in experimental animals. Acute high dosing regimens are quite distinct from the chronic usage patterns found in many human METH abusers. Furthermore, such doses, especially in nonhuman primates, can result in unexpected death, which is unacceptable, especially when such deaths fail to accurately model effects of human usage. As a model of chronic human METH abuse we have developed a nonlethal chronic METH administration procedure for the rhesus macaque that utilizes an escalating dose protocol. This protocol slowly increases the METH dosage from 0.1 to $0.7 \mathrm{mg} / \mathrm{kg}$ b.i.d. over a period of 4 weeks, followed by a period of chronic METH administration at $0.75 \mathrm{mg} / \mathrm{kg}$ b.i.d. ( = total daily METH administration of $1.5 \mathrm{mg} / \mathrm{kg}$ ). In parallel to human usage patterns, METH injections were given 20-23 times a month. This regimen produced a number of behavioral and physiological effects including decreased food intake and a significant increase in urinary cortisol excretion.

Neuropsychopharmacology (2005) 30, 350-359, advance online publication, I 3 October 2004; doi: I 0. I 038/sj.npp. I 300575
\end{abstract}

Keywords: drug abuse; animal models; amphetamines; methamphetamine; cortisol; temperature

\section{INTRODUCTION}

Methamphetamine (METH) abuse can induce neuronal dysfunction and CNS pathology, often in subcortical regions of the brain (Seiden and Ricaurte, 1987; Woolverton et al, 1989; Heaton et al, 1995; Hall et al, 1996; Kieburtz et al, 1996). To date, most studies involving METH administration and the consequent CNS pathology have focused on neurotoxicity in the striatal dopamine projections (Ricaurte et al, 1984; Cadet et al, 1998; Villemagne et al, 1998; Harvey et al, 2000b). However, the applicability of these studies to human METH abuse has been questioned due to differences between the pattern of drug-abuse dosage/frequency in human users and those used in animal studies. This concern has been addressed by only a few studies, including a recent report revealing that the gradual escalation of METH dosing alters both the physiological and behavioral responses to a subsequent high dose METH exposure in rodents (Segal et al, 2003).

*Correspondence: Dr HS Fox, Neuropharmacology, CVN-I, The Scripps Research Institute, 10550 N. Torrey Pines Rd, La Jolla, CA 92037, USA, Tel: + I 858784 7I7I, Fax: + I 858784 7296,

E-mail: hsfox@scripps.edu

Received 3 November 2003; revised 9 July 2003; accepted 19 August 2004

Online publication: 23 August 2004 at http://www.acnp.org/citations/ Npp082304035 I0/default.pdf
Unlike human chronic METH abuse patterns, most animal studies use high dose acute METH administration to examine toxic effects on the brain, frequently in striatal dopaminergic projections. High dose acute METH administration is often implemented because it offers the most consistent evidence of METH-induced CNS pathology. In rodents, one of the most common acute high dose METH administration protocols used is $10 \mathrm{mg} / \mathrm{kg}$ injected four to five times over the course of a single day at $2 \mathrm{~h}$ intervals (Broening et al, 1997; Chapman et al, 2001; Gluck et al, 2001), although doses as high as $40 \mathrm{mg} / \mathrm{kg}$ are also administered (Sabol et al, 2001). Short-term protocols are often utilized as well in nonhuman primate studies, with METH doses ranging from 0.5 to $2 \mathrm{mg} / \mathrm{kg}$ given for just $1-5$ days, two to four times each day (Melega et al, 1997; Villemagne et al, 1998; Harvey et al, 2000a,b). However, such dosing can lead to severe symptoms of METH toxicity resulting in the stoppage of the scheduled dosing regimen or even death of experimental animals. Through the use of relatively continuous escalating dosing (every $3 \mathrm{~h}$ around the clock) it has been shown that tolerance can develop in rhesus monkeys, at least to the anorectic and lethal effects of METH (Fischman and Schuster, 1974). In order to model chronic METH exposure in animal studies in a manner similar to human use patterns, development of tolerance to the potentially lethal effects of METH is required. The major lethal effect of METH exposure in animal studies is the 
development of hyperthermia. Therefore, the purpose of the present study was to develop a safe escalating dosing regimen that did not produce potentially lethal hyperthermia while simultaneously producing stimulant effects similar to those seen in human METH abuse such as reduced food intake and behavioral changes. Such a model will allow for the examination of the effects of chronic METH exposure on both physiology and behavior, as well as the interaction of chronic METH exposure with other disease processes.

Due to the inaccuracies commonly associated with subjective self-reports and the variability in purity of street drugs, exact dose/frequency patterns are difficult to determine in recreational METH users (Angrist, 1994; Huber et al, 1997; Simon et al, 2002). METH abusers appear to vary widely in their dosage/frequency of use as suggested by self-report and post-mortem drug testing. Such variation reflects the fact that drug users in general are fairly heterogeneous. Studies suggest that the doses of METH used by humans recreationally or in medical studies range from 5 to $1000 \mathrm{mg}$ over a $24 \mathrm{~h}$ period (Mitler et al, 1993; Simon et al, 2002). Post-mortem drug testing in a forensic study revealed a large range in the blood METH concentration among decedents (Logan et al, 1998). In this study low post-mortem METH concentrations were frequently observed, with the highest concentrations occurring in only a small subset of the decedents where METH was determined to be the direct cause of death, suggesting that lower doses are commonly used. In a study of intravenous METH users in San Antonio, Texas, the most common usage pattern was two to three injections per day on 1-2 days per week (Zule and Desmond, 1999). Examination of METH drug use among arrestees in five Western US cities revealed that METH users ranged from an average (in each city) of 10.4-15.8 days of METH over the month before arrest (Pennell et al, 1999). A study of METH users in Los Angeles and San Bernardino Counties in California, the majority of whom were enrolled in treatment programs, revealed that $70 \%$ used METH more than 20 times per month, with METH commonly administered one to three times a day (Simon et al, 2002). Many users thus take METH on an intermittent, yet regular, basis.

Acute high dose METH administration protocols provide an adequate model for studying acute overdose pathologies, but the vast majority of human METH abusers do not suffer from such complications, which can include cardiovascular events, rhabdomyolysis, hyperthermia, and convulsions (Richards et al, 1999; NIDA, 2002). Chronic users can suffer from behavioral, psychiatric, and medical abnormalities, but even in those without obvious symptoms there is increasing concern over the long-term effect of METH exposure on the brain (NIDA, 2002). Furthermore, there are little data on the effects of such usage on many other physiological systems. Thus the development of a model of chronic METH exposure in nonhuman primates is timely for our understanding of this ongoing and worsening problem.

Specific dosing regimens are therefore needed to advance our understanding of CNS pathologies in long-term low/ moderate dose METH users. Further, unless it is the intent of the researcher to examine such, unexpected deaths due to acute toxicity should be avoided given the ethics, regulations and costs involved in the use of nonhuman primates in scientific research. For these reasons, the purpose of this study was to: (1) develop a pattern of METH exposure that models chronic low/moderate dose human usage patterns; (2) develop a pattern of METH exposure in the nonhuman primate that is safe enough to avoid unplanned deaths related to acute METH toxicity, thus allowing chronic dosing and long-term studies. The development of this model will enhance our understanding of how chronic administration of METH may alter a number of behavioral and physiological parameters.

Relatively low single doses of METH in rhesus monkeys $(0.10-1.00 \mathrm{mg} / \mathrm{kg})$ are capable of inducing significant release of dopamine from the striatum (Tsukada et al, 2002); dopamine release is related to the euphoric effects of METH. Doses of METH taken during recreational use in humans is estimated at $20-40 \mathrm{mg}$, which for a $60-80 \mathrm{~kg}$ person equates to a $0.25-0.67 \mathrm{mg} / \mathrm{kg}$ dose (Jaffe, 1985), and an even lower dose ( $5 \mathrm{mg} ; 0.06-0.08 \mathrm{mg} / \mathrm{kg}$ ) is capable of resulting in positive drug effects in subjects (Hart et al, 2001). Therefore, in this study, we began with relatively low $(0.10 \mathrm{mg} / \mathrm{kg})$ doses of METH twice daily (b.i.d.), and then slowly escalated the dose over the next 4 weeks (METH Rampup) in an attempt to acclimate, or otherwise diminish, the animals' response to some of the untoward actions of the drug, such as sympathomimetic and hyperthermic effects. Animals were then injected with $0.75 \mathrm{mg} / \mathrm{kg}$ of METH b.i.d. (METH Maintenance), to mimic a pattern of chronic usage similar to many human users. Further, METH injections were given 5 days a week followed by 2 days of abstinence in order to model the periods of drug use followed by periods of abstinence reported by many METH users. We examined the effects of this regimen a number of physiological and behavioral parameters, including food intake and cortisol excretion. Here we present the results obtained through the METH Ramp-up and first 4 weeks of METH Maintenance.

\section{METHODS}

\section{Animals}

Using a within-subjects design, four male 3-5-year-old Rhesus macaques, weighing $4-7 \mathrm{~kg}$, free from SIV, type D simian retrovirus, and herpes $\mathrm{B}$ virus were obtained from Covance (Alice, TX) and Charles River (Key Lois, FL). Animals were kept in a biocontainment facility and housed individually in stainless-steel cages, which permit olfactory, visual, and auditory contact with other monkeys in the room. The room was maintained on a $12 / 12 \mathrm{~h}$ light/dark cycle (lights on 0600; lights off 1800) and at a temperature of $25.0^{\circ} \mathrm{C}$. All procedures were performed with approval of The Scripps Research Institute's Institutional Animal Care and Use Committee consistent with National Academy of Sciences Guide for Animal Care and Use (1996).

\section{Drug Treatment}

In order to acclimate all animals to the injection regimen and reduce stress, as well as to provide a within-subjects control period, each animal was injected intramuscularly (IM) with sterile $0.9 \%$ sodium chloride (Abbott Laboratories, North Chicago, IL) twice daily at 1030 and 1330 for 4 weeks (Saline). We used D-METH hydrochloride 
(Methamphetamine) (Sigma, St Louis, MO) diluted in sterile $0.9 \%$ sodium chloride to a dilution of $0.1-0.75 \mathrm{mg} / 0.1 \mathrm{ml}$. METH injections were given IM also twice daily in gradually escalating doses over 4 weeks (METH Ramp-up). After reaching the METH dose of $0.75 \mathrm{mg} / \mathrm{kgb.i.d}$. (METH Maintenance) the animals were maintained at this dose for the length of the study. On Fridays animals were given only one injection at 1030. When blood draws/CSF taps were scheduled (Fridays only) no METH or saline injections were given. METH injections were not given on weekends in order to model the periods of abstinence seen in human METH users. The dose given on the first day following a period of abstinence was sometimes lowered as a precaution against untoward METH effects, and single doses of METH were given on the day preceding a period of abstinence to minimize the possibility of unexpected METH-related problems. A complete description of the drug schedule can be found in Table 1 .

\section{Physiological Monitoring}

Temperature was measured continuously as part of the experimental parameters and as a safety precaution since
METH-induced hyperthermia has been shown to be lethal in nontolerant human drug users and laboratory animals (Davidson et al, 2001). Animals were implanted with telemetry transmitters (10TA-D70) capable of detecting body temperature (Data Sciences International, St Paul, MN) using aseptic surgical techniques under anesthesia. Transmitters were implanted into a subcutaneous pocket in the left flank by making a transverse incision $2 \mathrm{~cm}$ caudal to the left scapula. After device implantation, the incision was closed using 3.0-polyglactin sutures. Data acquisition was achieved through Data Sciences hardware (Receiver plate RLA 2000, St Paul, MN) and software (Dataquest A.R.T. Version 2, St Paul, MN). Data sampling occurred every $10 \mathrm{~s}$ but was averaged into $10 \mathrm{~min}$ bins. The daytime averages of these $10 \mathrm{~min}$ bins were used for analysis. Animals were given 4 weeks to recover from surgery before beginning the experimental protocol.

\section{Urine Sampling}

Urine samples were collected into 21 expandable containers containing $20 \mathrm{~g}$ boric acid (Sigma, St Louis, MO) as a preservative on ice. Urine collections were setup at 1000

Table I METH Administration Protocol Used in this Study

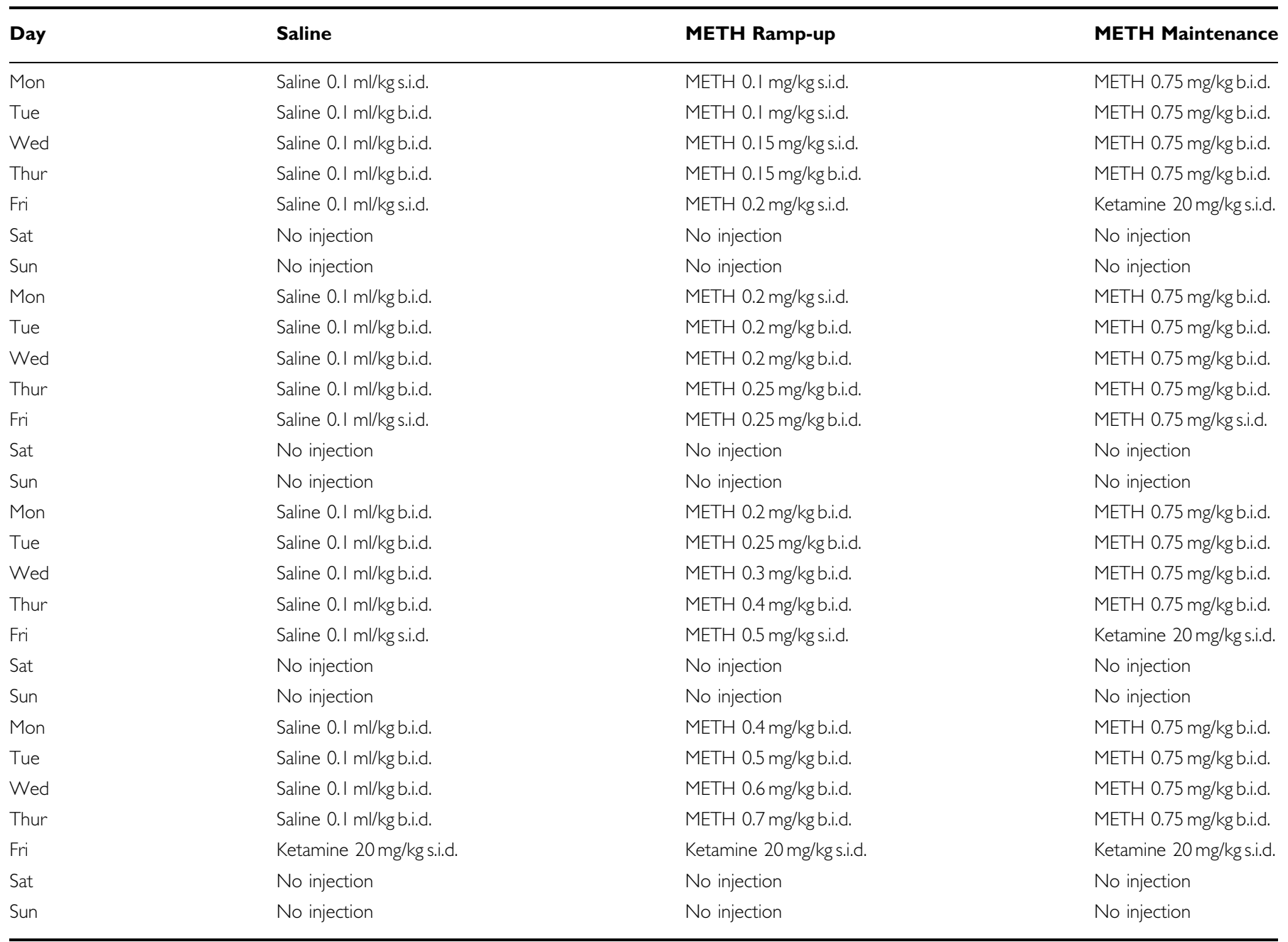

A gradual escalation of METH dose, from 0.1 to $0.7 \mathrm{mg} / \mathrm{kg}$, was given over the 4 weeks delineated as METH Ramp-up. A METH dose of $0.75 \mathrm{mg} / \mathrm{kg}$ b.i.d. was then continued throughout the period delineated as METH Maintenance. 
starting on Monday. At 1000 Tuesday-Friday, $24 \mathrm{~h}$ urine samples were collected. Total urine volume over the $24 \mathrm{~h}$ period was measured and $10 \mathrm{ml}$ aliquots were taken for later analysis. All samples were frozen at $-80^{\circ} \mathrm{C}$ until the time of assay.

\section{Food Intake}

Food intake (Purina Monkey Chow PMI \#5038) was measured daily at 1000 . Food was weighed prior to being placed in the cage and any food remaining in the hopper or tray the next day was weighed after drying (if wet from water, urine, or feces) and subtracted from the total given, this value was then used for analysis. To prevent animals from throwing uneaten food on the floor where it would be difficult to ascertain which monkey dropped it, all holes large enough for chow to be dropped through were covered.

\section{Cortisol Measurement}

Urine samples were thawed on ice and adjusted to a $\mathrm{pH}$ of 7.2 when not already in this range using $1 \mathrm{~N} \mathrm{HCl}$ or $1 \mathrm{~N}$ $\mathrm{NaOH}$ depending on the direction of change needed. Cortisol was measured in urine by ELISA using the standard protocol supplied with the kit (KMI Diagnostics, Minneapolis, MN). Calculation of $24 \mathrm{~h}$ urinary cortisol excretion was performed by multiplying each individual ELISA result ( $\mu \mathrm{g}$ per sample) against the $24 \mathrm{~h}$ urine volume $(\mathrm{ml})$ matching that sample (corrections were made for the amount of urine used in each ELISA well) to give the total number $\mu \mathrm{g}$ of cortisol excreted in $24 \mathrm{~h}$ for each sample day.

\section{METH Measurement}

All chemicals for the urine extractions were purchased from Sigma Aldrich (St Louis, MO, USA) with the exception of the HPLC grade water and $\mathrm{MeOH}$ that were from Burdick and Jackson (Muskegon, MI, USA). Pholedrine and pseudoephedrine were purchased from Sigma Aldrich (St Louis, MO, USA). METH, amphetamine (AMPH) and MDMA standards were provided by NIDA.

METH and its metabolite AMPH were extracted from $1 \mathrm{ml}$ volumes of selected urine samples utilizing a procedure adapted from Li et al (1997) using MDMA as an internal standard to account for extraction efficiency. Subsequently the content of METH, AMPH, and MDMA in each sample was determined using reversed phase gradient HPLC coupled with positive ion ESI-mass spectrometry (each sample was reconstituted with $100 \mu \mathrm{l}$ water containing $0.1 \mathrm{nmol}$ of pseudoephedrine as an internal standard for the LC-MS analysis). All analytes were detected in their $\mathrm{M}+\mathrm{H}$ charge state and were detected using selected ion monitoring of the appropriate mass/charge $(\mathrm{m} / \mathrm{z})$. External calibration curves were constructed daily for the estimation of sample analyte and internal standard concentrations. The limit of quantitation for each analyte was below $50 \mathrm{fg}$ on column (more than 50-fold lower than the lowest analyte concentration detected in this study). Calculation of $24 \mathrm{~h}$ urinary excretion of METH and its metabolites was performed by multiplying each $24 \mathrm{~h}$ urine volume against the mass spectrometry results for each sample.

\section{Statistical Analysis}

After determining data normality and homogeneity of the variances, statistical analyses were performed on either raw or ranked data using repeated measures one-way ANOVA with significance set at the $P<0.05$ level. Significant main effects were analyzed using the Dunnett or Dunns post hoc test. All of the above statistical tests were run using the software programs SigmaStat (SPSS Science, IL). Data are reported as mean \pm SEM.

\section{RESULTS}

\section{Temperature}

In this study, temperature remained relatively unchanged during the saline injection period (Figure 1). During the METH Ramp-up period alterations in temperature, which did not reach statistical significance, began to be observed. While temperature increased only slightly during the first week of the METH Ramp-up (0.1-0.15 mg/kg b.i.d.), weekend (no injection) temperatures dropped by $\sim 1{ }^{\circ} \mathrm{C}$ compared to the previous 5 days of METH administration, and by $\sim 0.5-0.7^{\circ} \mathrm{C}$ when compared to the 4 weeks of the Saline period. This pattern continued throughout the Ramp-up period, although larger, but still nonsignificant, increases in temperature were observed when the METH dose reached $0.4-0.5 \mathrm{mg} / \mathrm{kg}$ b.i.d.

During the first week of METH Maintenance, there was a trend towards slightly increased temperature, $\sim 0.3-0.4^{\circ} \mathrm{C}$ compared to saline, and this increase continued throughout the weekend (Figure 1). The pattern of temperature change during weeks 2-4 of METH Maintenance was similar to the pattern observed during the Ramp-up period, with higher temperatures early in the week and lower temperatures later in the week (ie lower temperature Friday compared to Monday), dropping $\sim 1^{\circ} \mathrm{C}$ on the weekends when METH was not administered. Still, a comparison of the METH Ramp-up and METH Maintenance periods against the Saline period showed no statistically significant change in temperature (Figure 1).

\section{Food Intake}

Average $24 \mathrm{~h}$ food intake over the 4 week Saline period was $190 \mathrm{~g}$ (Figure 2). During the early period of the METH Rampup, food intake dropped only slightly ( $\sim 8 \%$ change from the Saline period) or remained unchanged, but when METH levels reached $0.4 \mathrm{mg} / \mathrm{kg}$ b.i.d. or higher food intake decreased dramatically $(\sim 25-35 \%$ change from the Saline period; Figure 2). During the first 2 weeks of METH Maintenance food intake increased slightly, but decreased again during weeks 3 and 4 ( 10-30\% decrease from saline; Figure 2$)$. A comparison of average food intake during the METH Rampup and Maintenance periods to the Saline period revealed that food intake was significantly reduced following $\mathrm{METH}$ administration $(P<0.05$ for both comparisons).

\section{Cortisol}

Cortisol release is a major component of the mammalian response to stress. In humans, differences in the stress 


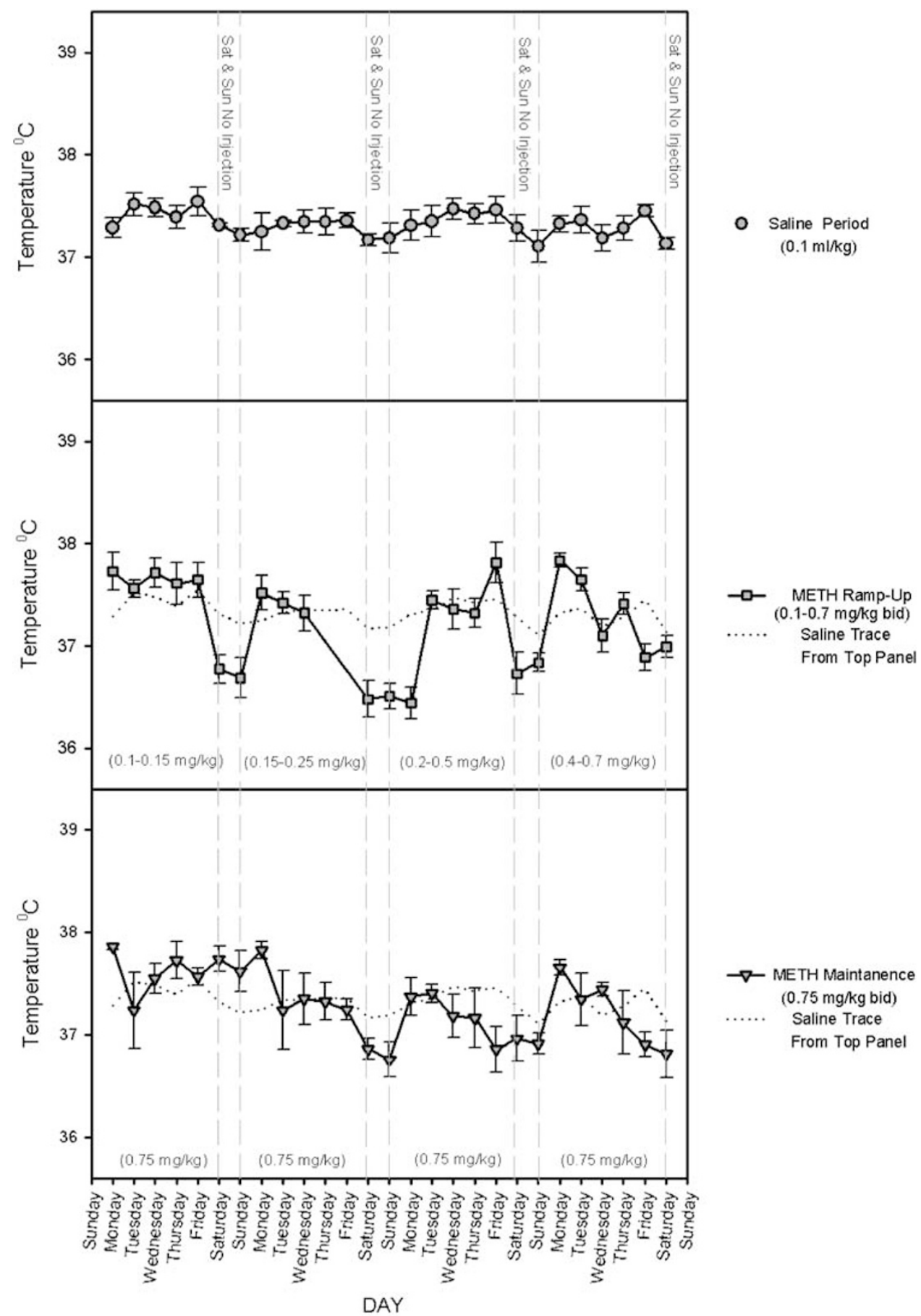

Figure I Effect of METH on average $24 \mathrm{~h}$ temperature over the course of all three periods, Saline, METH Ramp-up, and METH Maintenance (mean \pm SEM). There were no statistically significant changes in temperature following METH administration. In the two lower panels labeled METH Rampup and METH Maintenance the Saline period from the top panel is shown as a dotted line.

response have been implicated in drug abuse and amphetamine neurotoxicity (Miller and O'Callaghan, 1996; Soderpalm et al, 2003). We therefore measured total $24 \mathrm{~h}$ cortisol excretion in urine following METH administration. Saline injections alone produced very little alteration in total $24 \mathrm{~h}$ urinary cortisol excretion. METH administration on the other hand produced significant increases $(\sim 30-40 \%)$ in cortisol levels, both during METH Ramp-up and METH Maintenance periods when compared to the Saline period $(P<0.05$ for both comparisons; Figure 3$)$, with the largest increases in urinary cortisol excretion occurring during the METH Maintenance period.

\section{METH Levels}

Total $24 \mathrm{~h}$ urinary excretion of METH and its active metabolite AMPH was examined by mass spectrometry following the injection of the METH at doses of $0.25,0.50$, and $0.75 \mathrm{mg} / \mathrm{kg}$ b.i.d. at single time points during the METH Ramp-up and the METH Maintenance periods in order to 


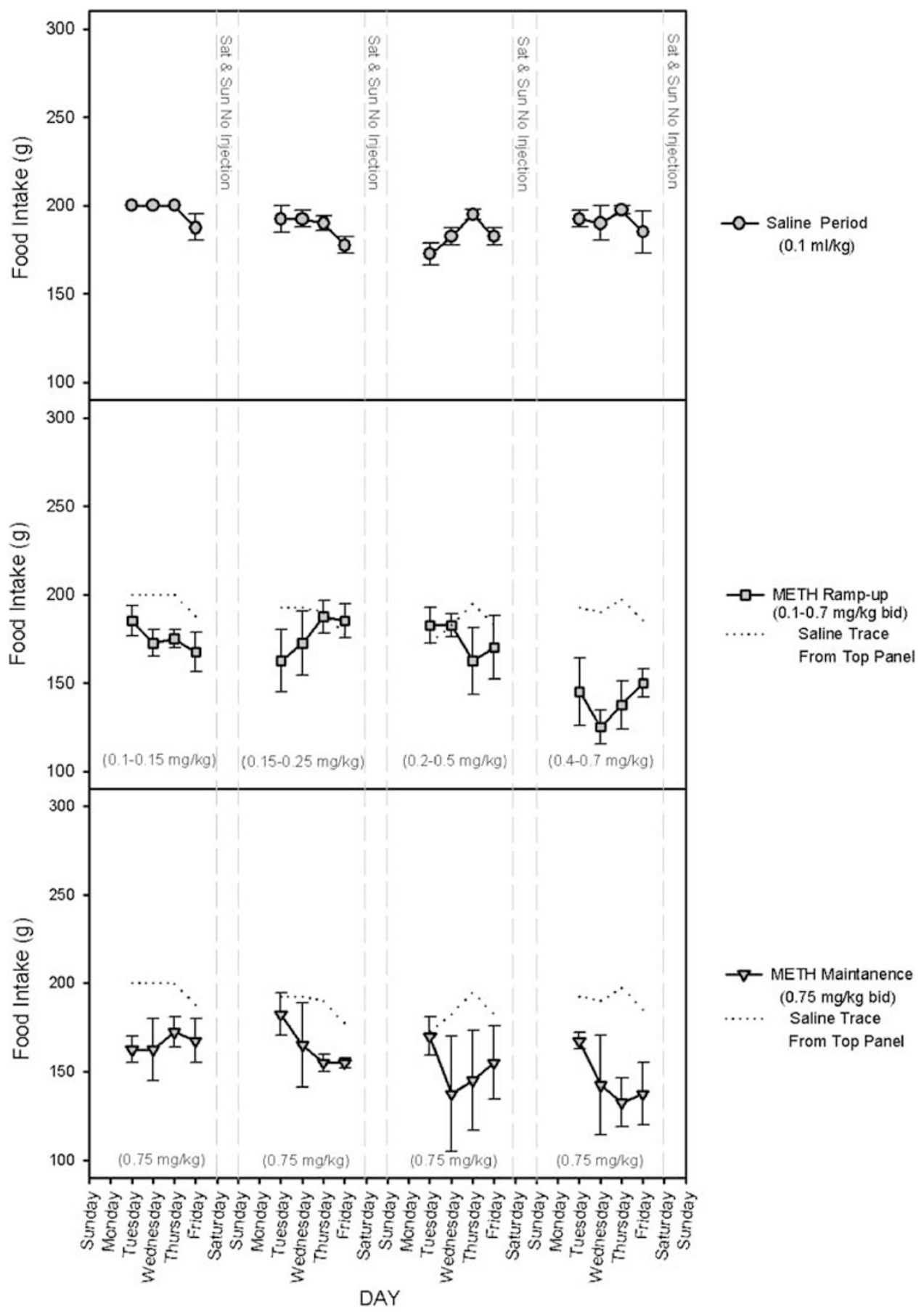

Figure 2 Effect of METH on average $24 \mathrm{~h}$ food intake over the course of all three periods, Saline, METH Ramp-up, and METH Maintenance (mean \pm SEM). There was a significant decrease food intake during both METH Ramp-up and METH Maintenance compared to the Saline period $(P<0.05)$. In the two lower panels labeled METH Ramp-up and METH Maintenance the Saline period from the top panel is shown as a dotted line.

assess the relative levels of active drugs during treatment. Excretion of METH occurred in a dose-dependent manner, although the changes appeared to be modest following each increase in the dose of METH administered (Figure 4).

\section{DISCUSSION}

Given the wide range in human dosage and frequency of METH administration, the goal of the study was to develop a safe, yet biologically relevant, METH administration protocol for chronic use in the nonhuman primate. To accomplish this we utilized a slowly escalating dosing regimen of METH as described in Table 1. The timing and the frequency of the drug administration was chosen based on the timing and frequency reported by many human METH users. Although human usage patterns vary greatly, we chose to give METH twice daily, starting in the morning for 5 days followed by 2 days of abstinence. This METH schedule is a realistic model for studying human drug use 


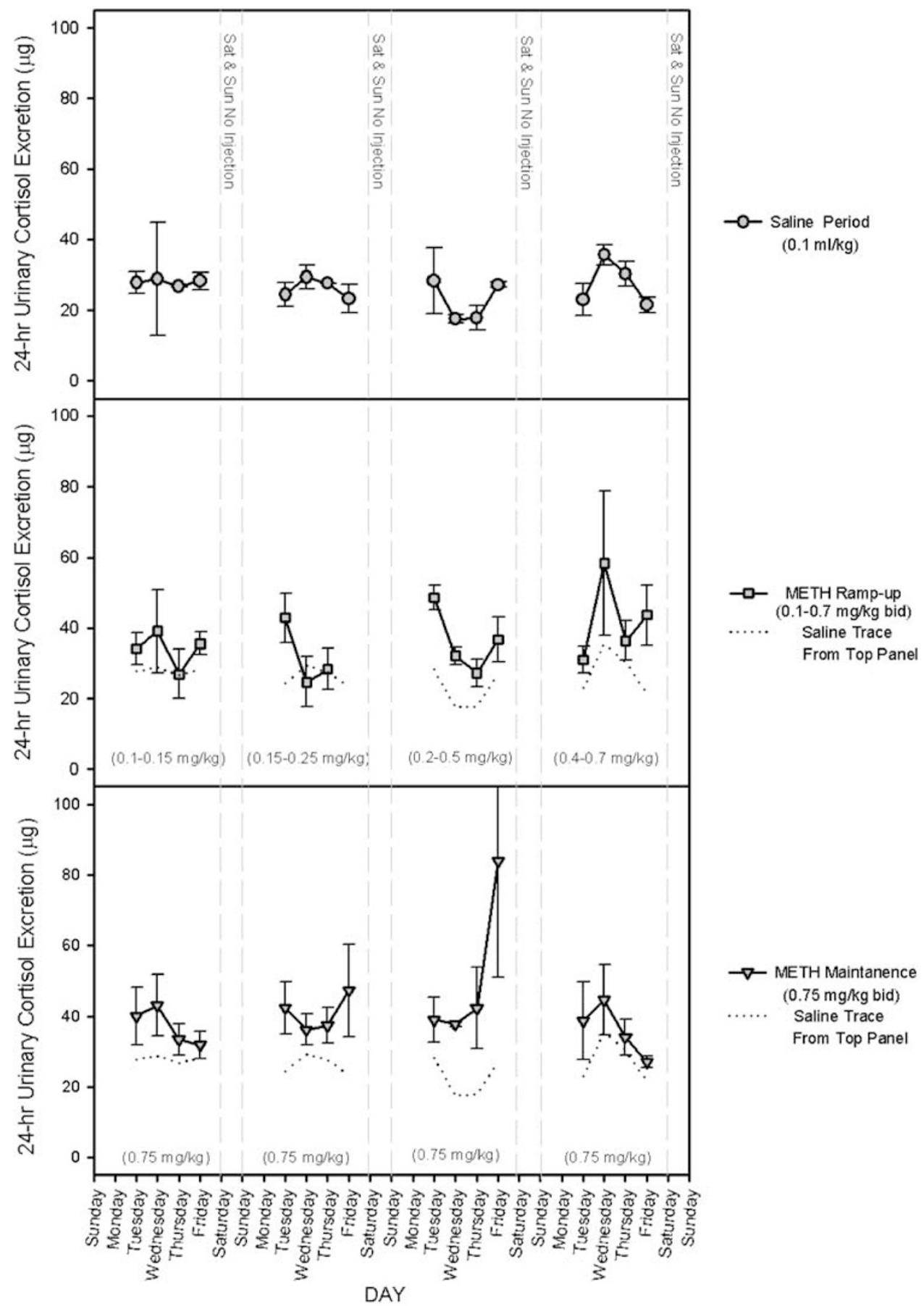

Figure 3 Effect of METH on average total $24 \mathrm{~h}$ urinary cortisol excretion over the course of all three periods, Saline, METH Ramp-up, and METH Maintenance (mean \pm SEM). There was a significant increase average $24 \mathrm{~h}$ urinary cortisol during both METH Ramp-up and METH Maintenance compared to the Saline period $(P<0.05)$. In the two lower panels labeled METH Ramp-up and METH Maintenance the Saline period from the top panel is shown as a dotted line.

since a considerable number human METH users report using one to three times a day during their normal waking hours, with the first dose being taken in the morning, with an average of $20+$ days of use per month, punctuated by periods of abstinence (Simon et al, 2002). Our dosing schedule exposed the animals to 20-23 days METH per month.

The drug dose administered during the METH Ramp-up period was chosen for safety reasons, known efficacy in inducing striatal dopamine release, and to mimic doses of drug taken by human METH users, in order to understand pathologies that may be related to chronic METH exposure. One of our main goals was to prevent critical hyperthermia in naïve animals, not only as a safety issue, but also because it has been implicated as cause of METH-related neurotoxicity in acute high dose protocols (Xie et al, 2000; Riddle et al, 2002; Brown et al, 2003). One of the long-term goals of this work is to understand how METH-induced hyperther- 


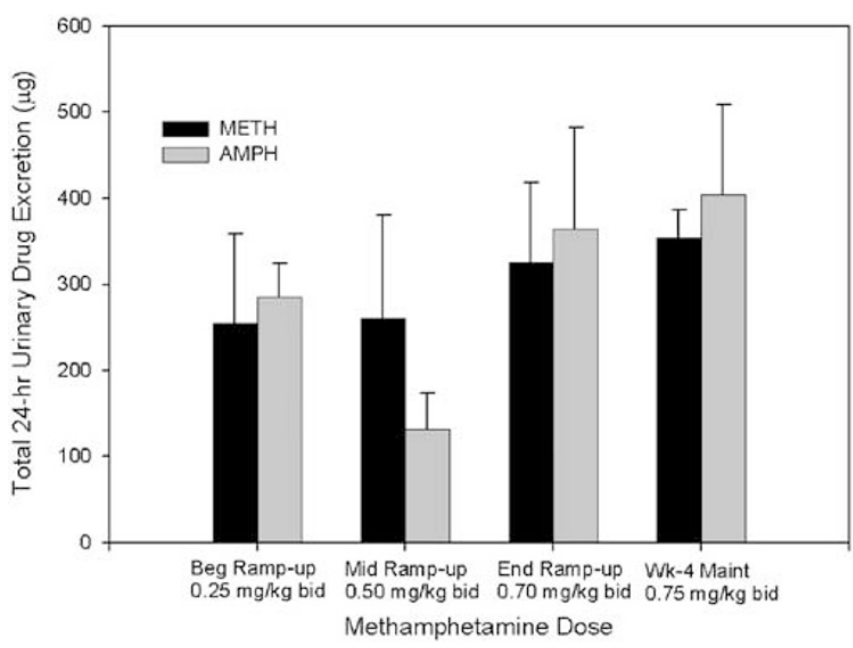

Figure 4 Average total $24 \mathrm{~h}$ urinary excretion of METH and its metabolite AMPH at selected single time-points during the METH Rampup and METH Maintenance periods (mean \pm SEM). Urinary METH excretion occurred in a dose-dependent manner although the changes appear to be very modest as the dose of METH administered increased. Urinary AMPH excretion was more variable.

mia may be disassociated experimentally from METH toxicity. To do this we utilized low doses of METH that gradually escalated over time. During the METH Ramp-up period escalation of the dose and frequency of the dosing were adjusted based on concerns for the animal's safety. Our dosing schedule errs on the side of caution, and it is likely that a more rapid or higher final METH dose could have been tolerated by the animals. Nevertheless, this model did meet our goal developing a safe METH protocol for the rhesus macaque.

Animal safety is a critical issue, particularly in nonhuman primate studies. Concern over unplanned deaths due to hypothermia in animal studies has been brought into the forefront following both published and unpublished studies. For example, researchers following the currently published METH dosing regimens are likely to see potentially lethal hyperthermia develop, at least in nonhuman primates. Administration of METH at a dosage of $2 \mathrm{mg} / \mathrm{kg}$, three times at $3 \mathrm{~h}$ intervals resulted in lethal malignant hyperthermia, along with other untoward effects, in one of five squirrel monkeys and one of five baboons in two studies (Ricaurte et al, 2002, 2003). Similarly, we observed toxic effects in our pilot studies in rhesus macaques using a similar dosing regimen of $2 \mathrm{mg} / \mathrm{kg}$ of $\mathrm{METH}$ injected IM four times every $2 \mathrm{~h}$ in one monkey, and a single daily dose beginning at $0.5 \mathrm{mg} / \mathrm{kg}$ and escalating to $3.5 \mathrm{mg} / \mathrm{kg}$ in a second monkey (unpublished observations). Our telemetric monitoring revealed that both animals developed critical hyperthermia following METH administration, requiring immediate veterinary intervention. While published reports do not list critical hyperthermia as a common side effect of METH use in humans, it is an issue in many animal studies, and therefore, requires significant attention if the researcher wishes to model the effects of chronic METH exposure.

Using the METH administration regimen described in Table 1, there was no evidence of critical hyperthermia or significant temperature changes occurring at any of the
METH doses employed in this study. The most robust, but once again statistically nonsignificant change in body temperature appeared to be the decrease in temperature on weekends. To fully understand this alteration, additional studies with more monkeys will be needed. However, this paradoxical lowering may be a drug withdrawal effect since METH was not given on weekends. Alterations in body temperature regulation during periods of abstinence have not been examined in human METH abusers, and could have important physiological and behavioral consequences. In contrast, large increases in body temperature are frequently reported in acute METH studies (Bowyer et al, 1992, 1994; Metzger et al, 2000).

While we achieved safety with the METH doses chosen for this study, we also acquired evidence to assess whether the amount of drug given was high enough to produce the desired psychostimulant state. Our measurements of food intake, as well as activity observations, were utilized to determine if our METH dosing schedule was capable of producing an altered psychological or physiological state. As the dose of METH increased, we observed significant decreases in food intake, an alteration that also occurs in humans using METH and other substituted amphetamines (Comer et al, 2001; Harris et al, 2002; Rawson et al, $2002 \mathrm{a}, \mathrm{b})$. The radiotelemetry system used in the present study allowed for the measurement of gross motor activity. However, radiotelemetric assessment of movement did not reveal differences in gross motor activity following METH administration. Still, informal visual observations performed during the study suggested increases in stereotypic behaviors (eg cage circling, excessive grooming, and nail biting) following METH injection. These behaviors were either absent or occurred at a very low-frequency preMETH or during the saline injection period. However, the behavioral observations were not conducted in a blinded manner over the entire course of the study and many of the stereotypic movements made by the animals were not picked up by the telemetric system measuring activity (gross movement are required for an activity count to be recorded), precluding our ability to make a quantitative assessment of METH's effects on activity in this study. We note that similar observations were reported in rhesus monkeys at METH doses between 0.5 and $1.25 \mathrm{mg} / \mathrm{kg}$, every $3 \mathrm{~h}$ (Fischman and Schuster, 1974). This behavior occurred for longer durations, perhaps due to the more frequent dosing. In any regard, such behavior is consistent in METHtreated rhesus monkeys.

Cortisol excretion was also dramatically altered by the doses of METH administered, particularly once the METH maintenance dose of $0.75 \mathrm{mg} / \mathrm{kg}$ b.i.d. was reached. To date, little is known about the effects of METH on cortisol release, a key element of the mammalian stress response. However, studies indicate that acute stress can modulate drug selfadministration and the perceived or physiological effects of drugs of abuse (Weiss et al, 2001; Marinelli and Piazza, 2002; Soderpalm et al, 2003). The relationship of cortisol to METH-induced pathology appears to be complex. Work with other substituted amphetamines in mice has shown that corticosterone supplementation or acute restraint stress diminishes hyperthermia and striatal neurotoxicity following drug administration (Miller and O'Callaghan, 1996). While hyperthermia is frequently observed following 
METH administration, in the present study, only small increases in temperature were observed, and we cannot determine whether the increased cortisol played a role in the lack of significant hyperthermia. It will be of interest in future studies to measure cortisol during the abstinence period. In doing so, we may be able to establish whether decreases in temperature are also accompanied by alterations in cortisol. While the study design did not allow for a full analysis of the relationship of cortisol to temperature or behavioral alterations, these results provide provocative implications for the involvement of the stress response in the physiological and behavioral responses to drug exposure, since cortisol excretion in this study varied significantly over the course of METH administration.

In summary, we have developed a chronic METH dosing model in rhesus monkeys that mimics human drug use patterns, with doses known to induce striatal dopamine release. METH administration by this protocol leads to physiological and behavioral alterations, such as increased cortisol excretion and decreased food intake. This slowly escalating drug administration regimen did not produce hyperthermia or death. Therefore, this model is safe for utilization in chronic nonhuman primate studies. In fact we have recently found that METH Maintenance can be continued for at least 7 months, and results in obvious neurochemical changes (LJM and HSF, in preparation).

METH abuse continues to grow in the US and elsewhere. The means by which METH leads to CNS damage with cognitive and psychiatric disorders (Cadet et al, 2003; Nordahl et al, 2003) the degree of potential neurochemical and functional recovery from damage (Volkow et al, 2001; Wang et al, 2004) and its interactions with other pathogenic challenges, such as HIV infection (Rippeth et al, 2004) are still relatively unknown. The use of this experimental animal model will enable the study of the chronic effects of METH, as well as therapeutic means for their amelioration.

\section{ACKNOWLEDGEMENTS}

We thank Debbie Watry and Dave Stouffer for technical assistance, and Nancy Delaney for manuscript preparation. This is manuscript \#15950-NP from The Scripps Research Institute. This work was supported by NIH grants nos. MH62261, MH61692, DA13390, DA12824, DA12444, and University of California AIDS Research Fellowship F02-SRI-209.

\section{REFERENCES}

Angrist B (1994). Ethical issues regarding prospective studies of amphetamine psychosis. J Calif Alliance Ment Ill 5: 32-35.

Bowyer JF, Davies DL, Schmued L, Broening HW, Newport GD, Slikker Jr W et al (1994). Further studies of the role of hyperthermia in methamphetamine neurotoxicity. J Pharmacol Exp Ther 268: 1571-1580.

Bowyer JF, Tank AW, Newport GD, Slikker Jr W, Ali SF, Holson RR (1992). The influence of environmental temperature on the transient effects of methamphetamine on dopamine levels and dopamine release in rat striatum. J Pharmacol Exp Ther 260: 817-824.
Broening HW, Pu C, Vorhees CV (1997). Methamphetamine selectively damages dopaminergic innervation to the nucleus accumbens core while sparing the shell. Synapse 27: 153-160.

Brown PL, Wise RA, Kiyatkin EA (2003). Brain hyperthermia is induced by methamphetamine and exacerbated by social interaction. J Neurosci 23: 3924-3929.

Cadet JL, Jayanthi S, Deng X (2003). Speed kills: cellular and molecular bases of methamphetamine-induced nerve terminal degeneration and neuronal apoptosis. FASEB J 17: 1775-1788.

Cadet JL, Ladenheim B, Hirata H (1998). Effects of toxic doses of methamphetamine (METH) on dopamine D1 receptors in the mouse brain. Brain Res 786: 240-242.

Chapman DE, Hanson GR, Kesner RP, Keefe KA (2001). Long-term changes in basal ganglia function after a neurotoxic regimen of methamphetamine. J Pharmacol Exp Ther 296: 520-527.

Comer SD, Hart CL, Ward AS, Haney M, Foltin RW, Fischman MW (2001). Effects of repeated oral methamphetamine administration in humans. Psychopharmacology (Berl) 155: 397-404.

Davidson C, Gow AJ, Lee TH, Ellinwood EH (2001). Methamphetamine neurotoxicity: necrotic and apoptotic mechanisms and relevance to human abuse and treatment. Brain Res Brain Res Rev 36: 1-22.

Fischman MW, Schuster CR (1974). Tolerance development to chronic methamphetamine intoxication in the rhesus monkey. Pharmacol Biochem Behav 2: 503-508.

Gluck MR, Moy LY, Jayatilleke E, Hogan KA, Manzino L, Sonsalla PK (2001). Parallel increases in lipid and protein oxidative markers in several mouse brain regions after methamphetamine treatment. J Neurochem 79: 152-160.

Hall M, Whaley R, Robertson K, Hamby S, Wilkins J, Hall C (1996). The correlation between neuropsychological and neuroanatomic changes over time in asymptomatic and symptomatic HIV-1infected individuals. Neurology 46: 1697-1702.

Harris DS, Baggott M, Mendelson JH, Mendelson JE, Jones RT (2002). Subjective and hormonal effects of 3,4-methylenedioxymethamphetamine (MDMA) in humans. Psychopharmacology (Berl) 162: 396-405.

Hart CL, Ward AS, Haney M, Foltin RW, Fischman MW (2001). Methamphetamine self-administration by humans. Psychopharmacology (Berl) 157: 75-81.

Harvey DC, Lacan G, Melegan WP (2000a). Regional heterogeneity of dopaminergic deficits in vervet monkey striatum and substantia nigra after methamphetamine exposure. Exp Brain Res 133: 349-358.

Harvey DC, Lacan G, Tanious SP, Melega WP (2000b). Recovery from methamphetamine induced long-term nigrostriatal dopaminergic deficits without substantia nigra cell loss. Brain Res 871: 259-270.

Heaton RK, Grant I, Butters N, White DA, Kirson D, Atkinson JH et al (1995). The HNRC 500-neuropsychology of HIV infection at different disease stages. HIV Neurobehavioral Research Center. J Int Neuropsychol Soc 1: 231-251.

Huber A, Ling W, Shoptaw S, Gulati V, Brethen P, Rawson R (1997). Integrating treatments for methamphetamine abuse: a psychosocial perspective. $J$ Addict Dis 16: 41-50.

Jaffe J (1985). Drug addiction and drug abuse. In: Goodman L, Gilman S (eds). Pharmacologicial Basis of Therapeutics. McMillan: New York. pp 284-324.

Kieburtz K, Ketonen L, Cox C, Grossman H, Holloway R, Booth H et al (1996). Cognitive performance and regional brain volume in human immunodeficiency virus type 1 infection. Arch Neurol 53: $155-158$.

Li NY, Li Y, Sellers EM (1997). An improved HPLC method for analysis of methamphetamine and its metabolites in plasma. Eur J Drug Metab Pharmacokinet 22: 427-432.

Logan BK, Fligner CL, Haddix T (1998). Cause and manner of death in fatalities involving methamphetamine. J Forensic Sci 43: $28-34$. 
Marinelli M, Piazza PV (2002). Interaction between glucocorticoid hormones, stress and psychostimulant drugs. Eur J Neurosci 16: 387-394.

Melega WP, Raleigh MJ, Stout DB, Lacan G, Huang SC, Phelps ME (1997). Recovery of striatal dopamine function after acute amphetamine- and methamphetamine-induced neurotoxicity in the vervet monkey. Brain Res 766: 113-120.

Metzger RR, Haughey HM, Wilkins DG, Gibb JW, Hanson GR, Fleckenstein AE (2000). Methamphetamine-induced rapid decrease in dopamine transporter function: role of dopamine and hyperthermia. J Pharmacol Exp Ther 295: 1077-1085.

Miller DB, O'Callaghan JP (1996). Neurotoxicity of D-amphetamine in the C57BL/6J and CD-1 mouse. Interactions with stress and the adrenal system. Ann NY Acad Sci 801: 148-167.

Mitler MM, Hajdukovic R, Erman MK (1993). Treatment of narcolepsy with methamphetamine. Sleep 16: 306-317.

NIDA (2002). Methamphetamine abuse and addiction, NIDA Research Report, NIH Publication \#02-4210.

Nordahl TE, Salo R, Leamon M (2003). Neuropsychological effects of chronic methamphetamine use on neurotransmitters and cognition: a review. J Neuropsychiatry Clin Neurosci 15: 317-325.

Pennell S, Ellett J, Rienick C, Grimes J (1999). Meth Matters: Report on Methamphetamine Users in Five Western Cities, National Institute of Justice, US Department of Justice, Research Report \#NCJ 176331.

Rawson RA, Anglin MD, Ling W (2002a). Will the methamphetamine problem go away? J Addict Dis 21: 5-19.

Rawson RA, Gonzales R, Brethen P (2002b). Treatment of methamphetamine use disorders: an update. J Subst Abuse Treat 23: $145-150$.

Ricaurte GA, Seiden LS, Schuster CR (1984). Further evidence that amphetamines produce long-lasting dopamine neurochemical deficits by destroying dopamine nerve fibers. Brain Res 303: 359-364.

Ricaurte GA, Yuan J, Hatzidimitriou G, Cord BJ, McCann UD (2002). Severe dopaminergic neurotoxicity in primates after a common recreational dose regimen of MDMA ('ecstasy'). Science 297: 2260-2263.

Ricaurte GA, Yuan J, Hatzidimitriou G, Cord BJ, McCann UD (2003). Retraction: severe dopaminergic neurotoxicity in primates after a common recreational dose regimen of MDMA ('ecstasy'). Science 301: 1479.

Richards JR, Johnson EB, Stark RW, Derlet RW (1999). Methamphetamine abuse and rhabdomyolysis in the ED: a 5-year study. Am J Emerg Med 17: 681-685.

Riddle EL, Kokoshka JM, Wilkins DG, Hanson GR, Fleckenstein AE (2002). Tolerance to the neurotoxic effects of methamphetamine in young rats. Eur J Pharmacol 435: 181-185.

Rippeth JD, Heaton RK, Carey CL, Marcotte TD, Moore DJ, Gonzalez R et al (2004). Methamphetamine dependence increases risk of neuropsychological impairment in HIV infected persons. J Int Neuropsychol Soc 10: 1-14.
Sabol KE, Roach JT, Broom SL, Ferreira C, Preau MM (2001). Long-term effects of a high-dose methamphetamine regimen on subsequent methamphetamine-induced dopamine release in vivo. Brain Res 892: 122-129.

Segal DS, Kuczenski R, O’Neil ML, Melega WP, Cho AK (2003). Escalating dose methamphetamine pretreatment alters the behavioral and neurochemical profiles associated with exposure to a high-dose methamphetamine binge. Neuropsychopharmacology 28: 1730-1740.

Seiden L, Ricaurte G (1987). Neurotoxicity of methamphetamine and related drugs. In: Meltzer HY (ed). Psychopharmacology: The Third Generation of Progress. Raven Press: New York. pp 359-366.

Simon SL, Richardson K, Dacey J, Glynn S, Domier CP, Rawson RA et al (2002). A comparison of patterns of methamphetamine and cocaine use. J Addict Dis 21: 35-44.

Soderpalm A, Nikolayev L, De Wit H (2003). Effects of stress on responses to methamphetamine in humans. Psychopharmacology (Berl) 170: 188-199.

Tsukada H, Miyasato K, Kakiuchi T, Nishiyama S, Harada N, Domino EF (2002). Comparative effects of methamphetamine and nicotine on the striatal $[(11) \mathrm{C}]$ raclopride binding in unanesthetized monkeys. Synapse 45: 207-212.

Villemagne V, Yuan J, Wong DF, Dannals RF, Hatzidimitriou G, Mathews WB et al (1998). Brain dopamine neurotoxicity in baboons treated with doses of methamphetamine comparable to those recreationally abused by humans: evidence from [11C]WIN-35,428 positron emission tomography studies and direct in vitro determinations. J Neurosci 18: 419-427.

Volkow ND, Chang L, Wang GJ, Fowler JS, Franceschi D, Sedler M et al (2001). Loss of dopamine transporters in methamphetamine abusers recovers with protracted abstinence. J Neurosci 21: 9414-9418.

Wang GJ, Volkow ND, Chang L, Miller E, Sedler M, Hitzemann R et al (2004). Partial recovery of brain metabolism in methamphetamine abusers after protracted abstinence. Am J Psychiatry 161: 242-248.

Weiss F, Ciccocioppo R, Parsons LH, Katner S, Liu X, Zorrilla EP et al (2001). Compulsive drug-seeking behavior and relapse. Neuroadaptation, stress, and conditioning factors. Ann NY Acad Sci 937: 1-26.

Woolverton WL, Ricaurte GA, Forno LS, Seiden LS (1989). Longterm effects of chronic methamphetamine administration in rhesus monkeys. Brain Res 486: 73-78.

Xie T, McCann UD, Kim S, Yuan J, Ricaurte GA (2000). Effect of temperature on dopamine transporter function and intracellular accumulation of methamphetamine: implications for methamphetamine-induced dopaminergic neurotoxicity. J Neurosci 20: $7838-7845$.

Zule W, Desmond D (1999). An ethnographic comparison of HIV risk behaviors among heroin and methamphetamine injectors. Am J Drug Alcohol Abuse 25: 1-23. 